\title{
FULL- AND HALF-GILBERT TESSELLATIONS WITH RECTANGULAR CELLS
}

\author{
JAMES BURRIDGE,* University of Portsmouth \\ RICHARD COWAN, ${ }^{* *}$ University of Sydney \\ ISAAC MA, ${ }^{* * *}$ Hong Kong Institute of Vocational Education
}

\begin{abstract}
We investigate the ray-length distributions for two different rectangular versions of Gilbert's tessellation (see Gilbert (1967)). In the full rectangular version, lines extend either horizontally (east- and west-growing rays) or vertically (north- and south-growing rays) from seed points which form a Poisson point process, each ray stopping when another ray is met. In the half rectangular version, east- and south-growing rays do not interact with west and north rays. For the half rectangular tessellation, we compute analytically, via recursion, a series expansion for the ray-length distribution, whilst, for the full rectangular version, we develop an accurate simulation technique, based in part on the stopping-set theory for Poisson processes (see Zuyev (1999)), to accomplish the same. We demonstrate the remarkable fact that plots of the two distributions appear to be identical when the intensity of seeds in the half model is twice that in the full model. In this paper we explore this coincidence, mindful of the fact that, for one model, our results are from a simulation (with inherent sampling error). We go on to develop further analytic theory for the half-Gilbert model using stopping-set ideas once again, with some novel features. Using our theory, we obtain exact expressions for the first and second moments of the ray length in the half-Gilbert model. For all practical purposes, these results can be applied to the full-Gilbert model—as much better approximations than those provided by Mackisack and Miles (1996).

Keywords: Random tessellation; point process; crack formation; fragmentation; division of space
\end{abstract}

2010 Mathematics Subject Classification: Primary 60D05; 05B45

Secondary $52 \mathrm{C} 17 ; 60 \mathrm{G} 55 ; 51 \mathrm{M} 20$

\section{Introduction}

Consider a stationary Poisson point process in the plane, of intensity $\lambda$. The particles of this process are called seeds, aptly so because at a given time $t=0$ they each initiate the growth of a line. The directions of the lines are randomly distributed, uniformly on $(0, \pi]$, and independent of each other and of the seed locations. Each line grows bidirectionally from its seed at the same rate; thus, two rays grow from each seed. When a ray encounters a line that has already grown across its path, the growth of that ray stops. Eventually, a tessellation of the plane is formed (see [9], where Schreiber and Soja provided a formal proof of convergence).

\footnotetext{
Received 3 November 2011; revision received 18 June 2012.

* Postal address: Department of Mathematics, University of Portsmouth, Portsmouth, UK.

Email address: james.burridge@gmail.com

** Postal address: School of Mathematics and Statistics, University of Sydney, NSW 2006, Australia.

Email address: rcowan@usyd.edu.au

*** Postal address: Hong Kong Institute of Vocational Education, 21 Yuen Wo Road, Sha Tin, Hong Kong.

Email address: isaacma@vtu.edu.hk
} 


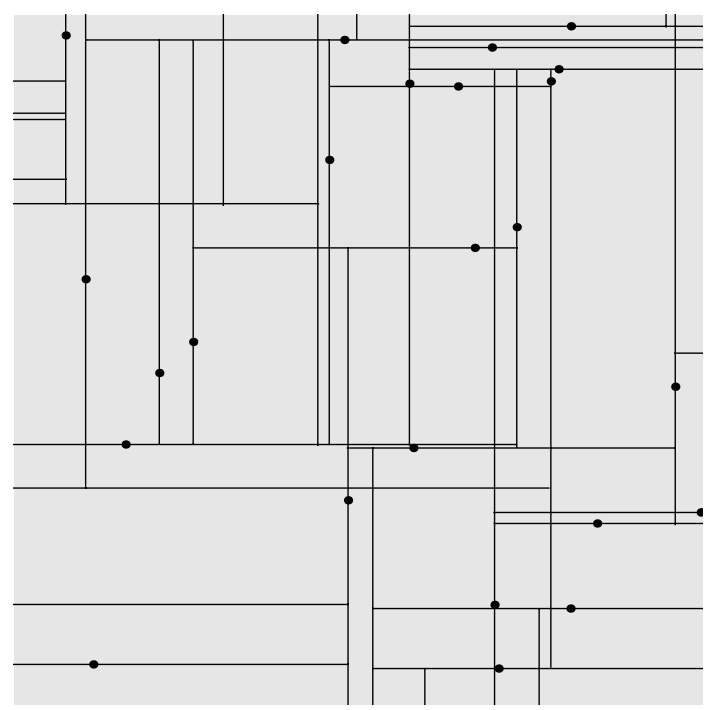

Figure 1: A realisation of the rectangular tessellation (discussed by Cowan and Ma) for the balanced case when V-type and $\mathrm{H}$-type seeds have equal intensities. A realisation of the full-Gilbert model having rectangular cells can be found in Figure 11 of [7].

The completed structure has become known as the Gilbert tessellation after Edgar N. Gilbert. It is notoriously difficult to analyse and even the expected length of a typical completed ray has not been found. There is no published paper by Gilbert on the topic; notes he supplied appear in a book (see [5]), with due acknowledgement to Gilbert. Citations have typically attributed the notes to Gilbert (as we do).

A version of the model where the directions of growth were confined to two orthogonal directions, vertical (V) and horizontal (H), was discussed by Mackisack and Miles [7]. A tessellation of the plane by rectangles results in their model. This structure too has not yielded to analysis, although when seeds are equally likely to be $\mathrm{V}$ or $\mathrm{H}$, the authors did provide an analytic approximation (based on ideas of Gilbert) to the expected ray length, namely, $\sqrt{2 / \lambda}$. The merits of this approximation have not been evaluated in the literature to date.

The current paper arises from work done in 1997 by the second and third authors (Cowan and Ma). They obtained some analytic results for an even simpler V\&H model, whereby the growth of eastward-growing rays is halted only by southward-growing rays (and vice versa). Westward and northward have the same reciprocity. A realisation of their tessellation is given in Figure 1.

For this model-which we call the half-Gilbert model because it has half of the blocking mechanisms - Cowan and Ma found a recurrence relationship (see (1) below) which they reported online [3], though without proof. The background to this recurrence is as follows.

Consider the isosceles right-angled triangle $P O Q$ in Figure 2(a). Here $|O P|=|P Q|=\ell$. Suppose that $n$ seeds lie inside the triangle, uniformly and independently distributed; $n=6$ in Figure 2(a). East or south growth of the rays is shown. Because of the blocking rules, only some of the rays reach the boundary of the triangle $P O Q$.

Cowan and Ma investigated the probability $h_{n}$ that no rays hit the boundary within the segment $O P$. This can also be interpreted as the probability that $L$, the final length of a test ray commencing eastward growth from $O$, is $>\ell$. 


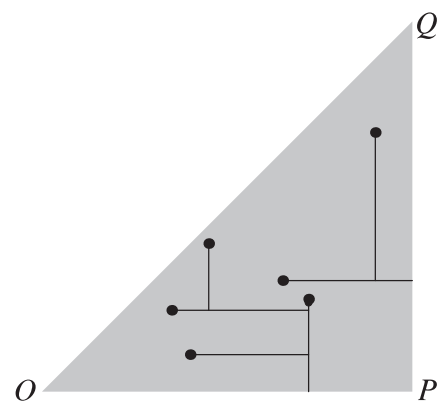

(a)

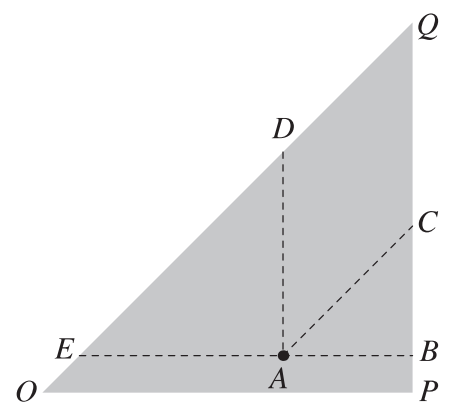

(b)

FIGURE 2: Diagrams to assist the proof of the Cowan-Ma recurrence.

Their recurrence relationship for $h_{n}$ was as follows. For $n \geq 1$,

$$
h_{n}=\frac{n ! q}{(2 n) !} \sum_{u=0}^{n-1} \sum_{v=0}^{n-1-u} \frac{2^{n-u-v} h_{u} h_{v}(n-1+u-v) !(n-1-u+v) !}{u ! v !(n-1-u-v) !}
$$

with $h_{0}=1$. Here $q$ is the proportion of seeds which grow horizontally. The recurrence does not involve $\ell$, so $h_{n}$ does not depend on $\ell$-as is obvious from the scale invariance of the problem posed by Figure 2(a).

This recurrence is a useful analytic step, providing precise information on $\mathbb{E}(L)$ and

$$
F(\ell):=\operatorname{Pr}\{L \leq \ell\}=1-\sum_{n \geq 0} h_{n} \frac{\left(\lambda \ell^{2}\right)^{n} \exp \left(-\lambda \ell^{2} / 2\right)}{2^{n} n !},
$$

from which we deduce (in an extended notation which includes $\lambda$ ) that $F_{\lambda}(\ell)=F_{1}(\sqrt{\lambda} \ell)$. Also,

$$
\begin{aligned}
\mathbb{E}(L) & =\int_{0}^{\infty}[1-F(\ell)] \mathrm{d} \ell \\
& =\sum_{n \geq 0} \frac{h_{n}}{n !} \int_{0}^{\infty}\left(\frac{\lambda \ell^{2}}{2}\right)^{n} \exp \left(-\frac{\lambda \ell^{2}}{2}\right) \mathrm{d} \ell \\
& =\frac{1}{\sqrt{2 \lambda}} \sum_{n \geq 0} \frac{h_{n} \Gamma(n+1 / 2)}{n !} .
\end{aligned}
$$

In Section 2 we report the proof used to derive the recurrence relationship (1) and plot the probability density function of the random variable $L$. The plot reveals the surprising property, first discovered when certain simulations of the full rectangular Gilbert model were plotted by Burridge [2], that the probability density function of the half rectangular Gilbert model with $\lambda=2$ is indistinguishable from that of the full rectangular Gilbert model with $\lambda=1$.

In Section 3 we present the simulation study, which has a very high level of accuracy, and discuss the abovementioned surprising property, which raises somewhat the profile of the halfGilbert model. As well as being of interest in its own right as a tessellation model with tractable mathematics, the model provides approximations for the full-Gilbert rectangular model. For example, the half-Gilbert model provides a much better approximation for $\mathbb{E}(L)$ in the full model when compared with the Mackisack/Miles' approximation $\mathbb{E}(L) \approx \sqrt{2 / \lambda}$ when $q=\frac{1}{2}$. 
In Section 4 our work demonstrates further the tractability of the half-Gilbert model; most notably, we find that the mean ray length when $q=\frac{1}{2}$ is given by

$$
\mathbb{E}(L)=\frac{\pi}{\sqrt{\lambda}(\Gamma(3 / 4))^{2}} .
$$

In both our simulation and analytical work we have employed Zuyev's concept of stoppingset sequences derived from Poisson point processes [10] and the distributional results for the areas of these sets. To achieve the analytic results, we have incorporated a new concept into the analysis, the idea of dead zones which influence the formation of the next stopping set in the sequence. Our most complete analysis is for the balanced case, $q=\frac{1}{2}$, because some results become rather complicated when $q \neq \frac{1}{2}$. The expected ray length in the latter case is reported, without proof, in Appendix A.

\section{The Cowan-Ma recurrence relation}

We now prove (1) for general $q$. Without loss of generality, given the remark above about the scale invariance of this problem, we set $\ell:=|O P|=1$.

Obviously, $h_{0}=1$ and $h_{1}=q$. When $n \geq 1$, we label the seed closest to $O P$ as $A$. See Figure 2(b). If the distance from $A$ to $O P$ is denoted by the random variable $Y$, it is easily shown that $Y$ has probability density function $g_{Y}(y)=2 n(1-y)^{2 n-1}, 0 \leq y \leq 1$. Furthermore, the conditional probability density function of $X:=|A B|$ given $Y$ is

$$
g(x \mid y)=\frac{1}{1-y}, \quad 0 \leq x \leq 1-y .
$$

Denote the event that no rays hit $O P$ by $\varepsilon_{n}$. Then

$$
\begin{aligned}
\operatorname{Pr}\left\{\varepsilon_{n} \mid x, y\right\}= & \operatorname{Pr}\{\text { seed } A \text { grows eastward and reaches } B \\
& \quad \text { and no ray grows across the segment } E A\} \\
= & q \operatorname{Pr}\{\text { no ray grows across } A B \text { and no ray grows across } E A\} .
\end{aligned}
$$

To evaluate the right-hand side, we partition the domain above $E B$ into the three zones that are shown in Figure 2(b). We then consider the trinomial distribution by which the remaining $n-1$ seeds are allocated to these zones: $u$ to $A B C, v$ to $E A D$, and the remaining $n-1-u-v$ to $A C Q D$. This leads, for each $(u, v)$, to a rather pleasing representation of the problem into two problems self-similar to the original problem. Continuing, using $\|\cdot\|$ as the area, we obtain

$$
\begin{aligned}
\operatorname{Pr}\left\{\mathcal{E}_{n} \mid x, y\right\}= & q \sum_{u=0}^{n-1} \sum_{v=0}^{n-1-u} \frac{(n-1) !\|A B C\|^{u}\|E A D\|^{v}\|A C Q D\|^{n-1-u-v}}{u ! v !(n-1-u-v) !\|E B Q\|^{n-1}} \\
& \times \operatorname{Pr}\{\text { no ray grows across } A B \text { and no ray grows across } E A \mid u, v\} \\
= & q \sum_{u=0}^{n-1} \sum_{v=0}^{n-1-u} \frac{(n-1) !\left(x^{2} / 2\right)^{u}\left((1-x-y)^{2} / 2\right)^{v}[x(1-x-y)]^{n-1-u-v}}{u ! v !(n-1-u-v) !\left((1-y)^{2} / 2\right)^{n-1}} \\
& \times \operatorname{Pr}\{\text { no ray grows across } A B \mid u\} \operatorname{Pr}\{\text { no ray grows across } E A \mid v\} \\
= & q \sum_{u=0}^{n-1} \sum_{v=0}^{n-1-u} \frac{(n-1) ! x^{2 u}(1-x-y)^{2 v}[2 x(1-x-y)]^{n-1-u-v}}{u ! v !(n-1-u-v) !(1-y)^{2(n-1)}} h_{u} h_{v} .
\end{aligned}
$$


Unconditional on $x$ and $y$, and with $n \geq 1$,

$$
\begin{aligned}
& h_{n}=\operatorname{Pr}\left\{\mathcal{E}_{n}\right\} \\
&=\int_{0}^{1} \int_{0}^{1-y} \operatorname{Pr}\{\mathcal{E} \mid x, y\} g_{Y}(y) g(x \mid y) \mathrm{d} x \mathrm{~d} y \\
&=q \sum_{u=0}^{n-1} \sum_{v=0}^{n-1-u} \frac{(n-1) ! h_{u} h_{v}}{u ! v !(n-1-u-v) !} \\
&=(n-1) ! q \sum_{u=0}^{n-1} \times 2 n \int_{0}^{1} \int_{0}^{1-y} x^{2 u}(1-x-y)^{2 v}[2 x(1-x-y)]^{n-1-u-v} \mathrm{~d} x \mathrm{~d} y \\
& u ! v !(n-1-u-v) ! \\
&= n ! q \sum_{u=0}^{n-1} \sum_{v=0}^{n-1-u} \frac{2^{n-1-u-v} h_{u} h_{v}}{u ! v !(n-1-u-v} h_{u} h_{v} \\
&=\frac{n ! q}{(2 n) !} \sum_{u=0}^{n-1} \sum_{v=0}^{n-1-u} \frac{2^{n-u-v} h_{u} h_{v}\left(n-1+u-v(1-y)^{2 n-1} B(n+u-v, n-u+v) \mathrm{d} y\right.}{u ! v !(n-1-u-v) !}(1-y)^{n-1-u+v} \mathrm{~d} x \mathrm{~d} y
\end{aligned}
$$

We augment this recurrence with the result $h_{0}=1$. Note that our expression of the double integral in (3) in terms of a single integral with beta function a line later follows from the substitution $x^{\prime}=w-y$ or by recognizing that (3) contains an Euler integral of the first kind. This completes the proof of (1). We note that the sequence $h_{0}, h_{1}, h_{2}, \ldots$ commences $1, \frac{1}{2}, \frac{1}{3}, \frac{29}{120}, \frac{11}{60}, \ldots$ when $q=\frac{1}{2}$.

Recurrence (1) together with (2) can be used to plot $f(\ell):=F^{\prime}(\ell)$ against $\ell$ for various values of $q$ (see Figure 3).

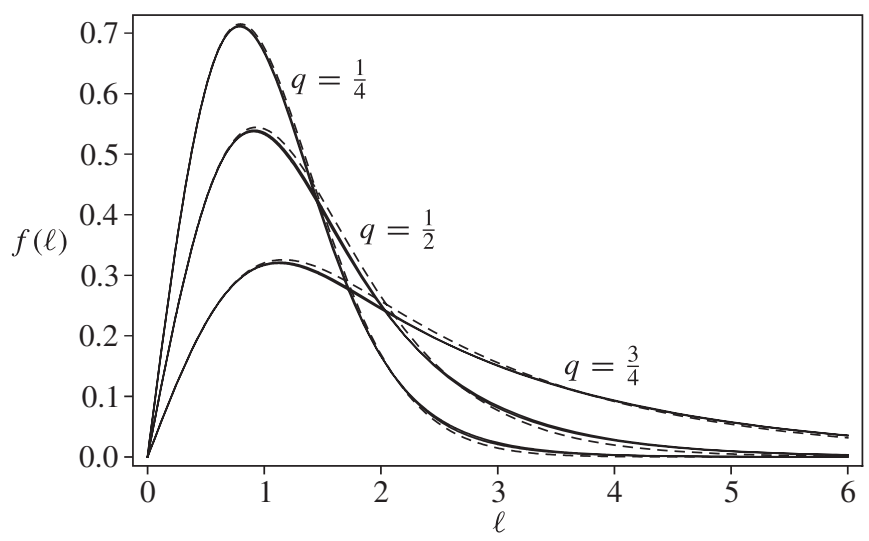

FIGURE 3: The solid curves are the probability density functions $f$ for the final length of a typical eastwardgrowing ray in the half-Gilbert model with $\lambda=2$ and $q=\frac{1}{4}, \frac{1}{2}, \frac{3}{4}$. Each solid curve actually comprises two curves overlaid, the second being the curve from the full-Gilbert model with $\lambda=1$. The dashed curves are the probability density functions from Gilbert's heuristic 'mean-field' analysis, also valid for both models. 


\section{Simulation of the full rectangular Gilbert tessellation}

Finding coefficients analogous to $h_{n}$ for the full rectangular model is a formidable task because of the complexity of the blocking effects. Lacking self-similar zones akin to those discovered by Cowan and Ma in their model, we have devised an efficient way of accounting for these effects by simulation.

The analogue of the isosceles triangle used in Figure 2 is a square, rotated so that its diagonal $A C$ lies east-west, as illustrated in Figure 4. To study the growth of horizontal rays, we consider an H-type test seed located at the western corner of the square, marked $A$ in the figure, and define

$$
\boldsymbol{h}_{n}=\operatorname{Pr}\{\text { ray from test seed } A \text { reaches } B \mid n \text { seeds in the square }\} .
$$

The only seeds that can block the test ray lie in the western side of the square, but whether or not they do so also depends on the configuration of seeds in the eastern side. Seeds outside the square have no influence.

By analogy with (2), the ray length distribution for the rectangular Gilbert tessellation is

$$
\boldsymbol{F}(\ell)=1-\sum_{n \geq 0} \boldsymbol{h}_{n} \frac{\left(2 \lambda \ell^{2}\right)^{n} \exp \left(-2 \lambda \ell^{2}\right)}{n !},
$$

from which $\boldsymbol{F}_{\lambda}(\ell)=\boldsymbol{F}_{1}(\sqrt{2 \lambda} \ell)$ is deduced.

\subsection{An obvious method}

The naive approach to estimating $\boldsymbol{h}_{n}$ would be to repeatedly populate the large square in Figure 4 with $n$ seeds (each independently of $\mathrm{H}$ type with probability $q$ ) and, each time,

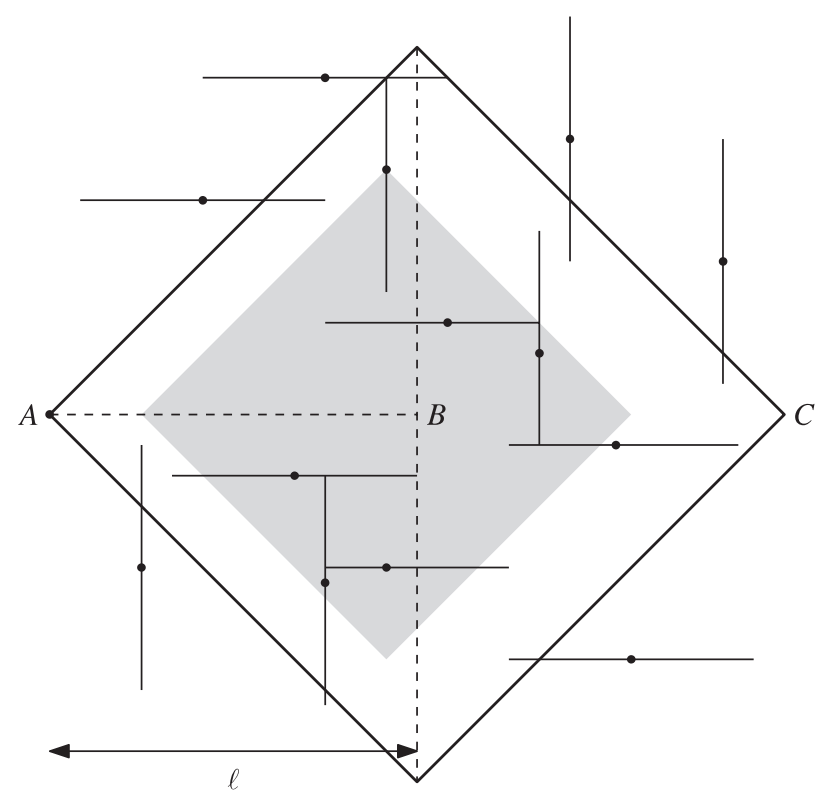

FIGURE 4: Only seeds within the large square (whose diagonal is $A C$ ) can influence the event that an H-type ray starting at $A$ does not reach $B$ (due to intersection of the line segment $A B$ by vertical rays).

The role of the smaller shaded square is described in the text. 
determine if the line $A B$ is intersected. This can be accomplished using the following recursive algorithm which decides if a ray, extending in compass direction $u \in\{\rightarrow, \uparrow, \leftarrow, \downarrow\}$, from one seed $s^{*}$ will be blocked within a distance $d$. The algorithm, block, outputs a logical value:

$$
\operatorname{block}\left(s^{*}, d, u\right)= \begin{cases}\text { true } & \text { if ray is blocked, } \\ \text { false } & \text { if ray is not blocked. }\end{cases}
$$

Whether or not $s^{*}$ is blocked within a distance $d$ depends only on the configuration of seeds within a square of diagonal $2 d$ along which its produced ray travels. Let the compass direction of this ray be $u$, and let us denote by $\Delta\left(s^{*}, d, u\right)$ the isosceles triangle which forms the half of the square closest to $s^{*}$. Let the type ( $\mathrm{H}$ or $\mathrm{V}$ ) of seed $s$ be $t(s)$. The algorithm $\operatorname{block}\left(s^{*}, d, u\right)$ runs as follows.

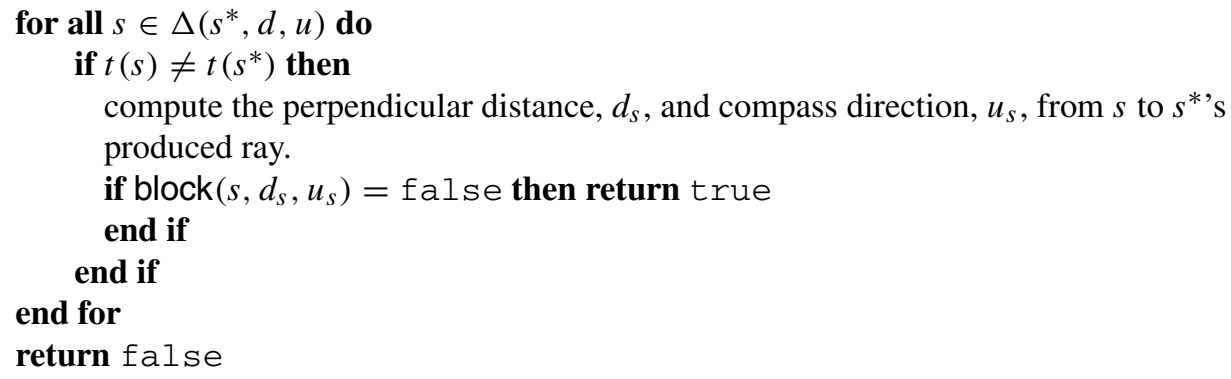

For example, if $s^{*}$ is the H-type seed at $A$ then the computer program calls block $\left(s^{*}, \ell, \rightarrow\right)$. This invokes recursive calls to block for every V-type seed in the left isosceles triangle (until a true value is returned by the call). In Figure 4, the shaded region with a V-type seed $s$ at the top shows a square that is investigated by one of the recursive calls, specifically by the call $\operatorname{block}\left(s, d_{s}, \downarrow\right)$, where $2 d_{s}$ is the diagonal length of the shaded square.

In principle, we can conduct this simulation for each $n$ up to (say) 300. For each $n$, we would generate the seeds in the square (with diagonal $A C$ ) $N$ times, where $N$ would be very large. An estimate of $\boldsymbol{h}_{n}, 0 \leq n \leq 300$, is thereby generated for H-type rays. Then, if $q \neq \frac{1}{2}$, we would repeat the whole procedure for V-type rays. It is an unnecessarily lengthy process, despite the potential saving if an early tested seed $s$ returns true, implying that others do not have to be tested.

\subsection{Stopping sets}

To shorten the task, we have devised a method based on stopping sets (a concept defined in the context of Poisson point processes by Zuyev [10] and developed further in [4]). Consider the unbounded quadrant that lies between the half-lines $y=x$ and $y=-x$, with $x \geq 0$, partly shown in Figure 5(a). A stationary Poisson process of seeds with intensity $\lambda$ exists in the quadrant. A triangular set whose eastern boundary is vertical and western vertex is the quadrant's apex is gradually expanded, stopping briefly whenever its boundary hits a seed (before continuing its expansion). The set stopped by the $k$ th seed encountered is called $S_{k}$. This process creates a nested sequence of random sets. We denote the area of $S_{1}$ by $E_{1}$ and the areas of the region $S_{k} \backslash S_{k-1}$ by $E_{k}, k>1$. Another nesting arrangement is shown in Figure 5(b), this time with squares and a different ordering of the seeds.

Zuyev showed, among other things of a more general nature, that any expanding domain constructing a nest of compact sets in the manner described above, through a sequence of stops caused by seed hits, creates areas $E_{1}, E_{2}, E_{3}, \ldots$ which are independent and distributed 


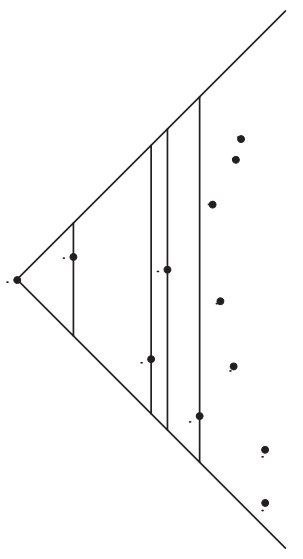

(a)

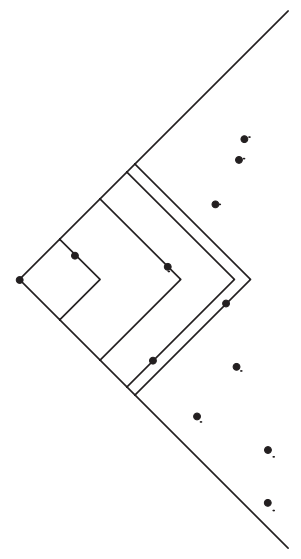

(b)

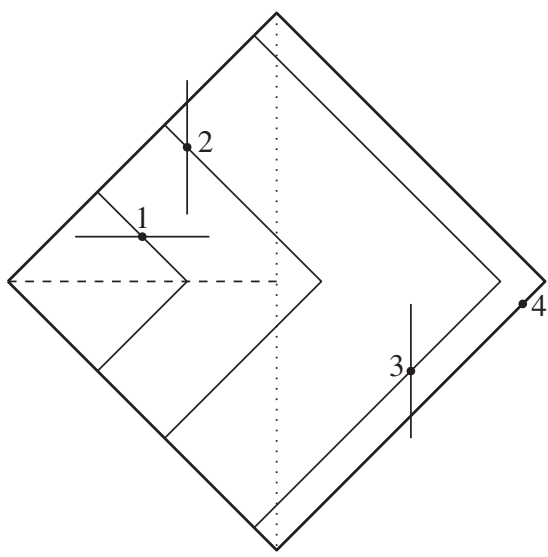

(c)

FiguRe 5: Nested stopping sets are shown in (a) and (b). In (c), the 'efficient algorithm' is at step 3 and at least one more step is needed before we see a ray in step $n$ crossing the dashed half-diagonal of $S_{n+1}$.

exponentially with parameter $\lambda$. The domain might have a complicated geometry because the expansion rule is allowed to depend on the seeds that it contains (and, being closed, this includes seeds on the domain's boundary). In the two examples of Figure 5, the expansion rule is straightforward and does not depend on the internal seeds.

Most importantly for the validity of Zuyev's distributional results, neither the expansion rule nor the stopping rule for $S_{1}$ should depend on seeds outside the expanding domain. This prohibition plays a twofold role:

(R1) it helps establish that $E_{1}$ is exponentially distributed;

(R2) it also allows one to say that the point process of seeds outside the stopping set $S_{1}$ is still a stationary Poisson point process with unchanged intensity given the information within $S_{1}$ (a notion formalized by Theorem 2 of [4]).

This allows the argument to be extended sequentially to $E_{2}, E_{3}, \ldots$ and $S_{2}, S_{3}, \ldots$

Remark 1. Another condition is also relevant for the (R1). Zuyev's theory in its general form requires a 'scale invariance' condition (defined in Equation (10) of [10]). In our context, this condition is trivially automatic because, for all $k, \operatorname{Pr}\left\{\right.$ our stopping set $S_{k}$ contains exactly one seed $\}=1$, a quantity which does not depend on $\lambda$ (as required in Zuyev's condition).

Remark 2. We also note that Zuyev's results are not guaranteed if randomisations apart from the Poisson process of seeds affect the growth and stopping. No such complication occurs in this section of our paper, although we must address the issue in Section 4.

Stopping sets $S_{1}, S_{2}, \ldots$ constructed in the way described above have other properties. The $i$ th seed $s_{i}$ is uniformly distributed on the growth frontier of $S_{i}$ and the seeds $s_{1}, s_{2}, \ldots, s_{n}$ are uniformly and independently distributed in the set $S_{n+1}$. Those of Figure 5(b) have a property that no other nesting has:

(P) if $s_{i}$ is $\mathrm{V}$ type then whether or not it reaches the east-west diagonal depends only on seeds $s_{1}, s_{2}, \ldots, s_{i-1}$. 


\subsection{Efficient algorithm}

In the context of Figure 5(b) with its nesting of squares, property (P) states that the ray growth just within $S_{n+1}$ from the seeds $s_{1}, s_{2}, \ldots, s_{n}$ provides a sample of the problem that interests us, giving a true or false datum on whether a test ray is blocked before it traverses half the diagonal of $S_{n+1}$. (See the illustration for $n=3$ in Figure 5(c).) This datum contributes to the estimation of $\boldsymbol{h}_{n}$. Importantly, as we show below, if the datum is true then we can add a true datum for the estimation of all $\boldsymbol{h}_{j}, j>n$, without further computational effort.

We start with the unbounded quadrant empty of seeds, then place an H-type test seed at the apex of the quadrant. We generate the exponentially distributed areas $E_{1}$ and $E_{2}$, and so construct the squares $S_{1}$ and $S_{2}$ expanding from the apex. We randomly select (uniformly) a seed point $s_{1}$ on the growth frontier (eastern sides) of the inner square, $S_{1}$. Because of the properties discussed above, this is equivalent to choosing the point uniformly within the outer square $S_{2}$. If this seed grows a vertical ray that intersects the diagonal, let the distance of the intersection point from the apex be $X_{1}$. If not, set $X_{1}=\infty$.

Let $A_{i}$ denote $\left\|S_{i}\right\|$, the area of $S_{i}$, and let $\varepsilon_{n}$ denote the event that the line from the test seed reaches the centre of a square populated with $n$ uniformly distributed seeds. Obviously, $\boldsymbol{h}_{n}=\operatorname{Pr}\left\{\boldsymbol{E}_{n}\right\}$.

If $X_{1}<\left(A_{2} / 2\right)^{1 / 2}$ then the simulation ends. There is no need to generate more nested squares in order to simulate the events $\varepsilon_{n}, n>1$, because we know that the half-diagonal of every subsequent square will be crossed at $X_{1}<\left(A_{n} / 2\right)^{1 / 2}$. Seeds on the boundaries of subsequent squares cannot influence this. If the first seed does not cross the diagonal, or crosses such that $X_{1}>\left(A_{2} / 2\right)^{1 / 2}$, then we draw $S_{3}$ and pick a point $s_{2}$ on the boundary of the second nested square $S_{2}$. We check whether $s_{2}$ 's ray intersects the diagonal, accounting for any possible blocking effects from $s_{1}$ by using the algorithm block. If so, we let the distance from the apex to the closest intersection point be $X_{2}$, which will be less than or equal to $X_{1}$. If $X_{2}<\left(A_{3} / 2\right)^{1 / 2}$ then the simulation ends. If not, we add another square $S_{4}$ and seed $s_{3}$, reaching the situation in Figure 5(c), and so on. We keep repeating the process, adding another seed and using block on that seed, until block indicates that the latest half-diagonal has been hit. We then record that the event $\varepsilon_{n}$ fails to occur for this and all higher values of $n$. The entity $\boldsymbol{h}_{n}$ for eastward-growing rays is the fraction of times that $\varepsilon_{n}$ occurs over many simulations. If $q \neq \frac{1}{2}$, the complete protocol is repeated with $q$ replaced by $1-q$ to give results for southward-growing rays.

To estimate the $\boldsymbol{h}_{n}, N=10^{9}$ simulations were performed, requiring a running time of approximately one hour on a modern PC (this being less than $1 \%$ of the time required for our 'obvious method' discussed above to achieve a similar precision). When $q=\frac{1}{2}$, the largest number of nested squares created before the simulation terminated was 917 , which occurred once, and the second largest number was 727, which also occurred once. The mean number of squares created before termination was 5.25. In the $q=\frac{1}{2}$ case, the estimate of the expected length of each line produced from a seed was

$$
\mathbb{E}(L)=1.467535(0.000029)
$$

where the bracketed number is the standard error, calculated with due regard to the positive covariance between our estimators $\boldsymbol{h}_{n}$ and $\boldsymbol{h}_{n+k}, k>0$.

Remark 3. Our accurate estimate of the $\boldsymbol{h}_{n}$ values allows the probability density function of the ray length to be calculated. Because the two ray lengths coming from a particular seed are independent, the standard convolution method leads to an estimated distribution of the total line length arising from a typical seed. Mackisack and Miles [7] claimed that these two ray lengths 
are not independent, but we disagree. As stated earlier in this section, seeds outside the square of Figure 4 do not influence the length of the east-growing ray. Likewise, a west-growing ray is not influenced by seeds outside a similar square drawn to the west of the test seed. These two squares, which intersect only at the starting position of the test seed, have no other seeds in common and have independent Poisson seedings in their interiors.

\subsection{The coincidence}

We found a remarkable similarity between the probability density functions in the half rectangular Gilbert model and the full rectangular Gilbert model when the intensity of seeds in the former case was twice that of the latter case. Indeed, the plots were almost indistinguishable, so Figure 3 effectively displays both $f$ and $f$ for various $q$, with $\lambda=2$ and $\lambda=1$, respectively.

We were mindful that the simulated results have sampling errors, albeit small. So we asked the question: are the two distributions $F$ and $\boldsymbol{F}$ mathematically equal, or just approximately so? To answer this in the $q=\frac{1}{2}$ case, we performed some rather tedious exact calculations (details omitted) which yielded

$$
\boldsymbol{h}_{0}=1, \quad \boldsymbol{h}_{1}=\frac{3}{4}, \quad \boldsymbol{h}_{2}=\frac{7}{12}, \quad \boldsymbol{h}_{3}=\frac{7}{15} .
$$

We then expanded both $F$ and $\boldsymbol{F}$ as a Taylor series about the origin:

$$
\begin{aligned}
F(\ell)= & h_{0}+\left(h_{1}-h_{0}\right) \ell^{2}+\frac{1}{2}\left(h_{0}+h_{2}-2 h_{1}\right) \ell^{4} \\
& +\frac{1}{6}\left(3 h_{1}-3 h_{2}-h_{0}+h_{3}\right) \ell^{6}+o\left(\ell^{7}\right) \\
= & 1-\frac{1}{2} \ell^{2}+\frac{1}{6} \ell^{4}-\frac{31}{720} \ell^{6}+o\left(\ell^{7}\right), \\
\boldsymbol{F}(\ell)= & \boldsymbol{h}_{0}+2\left(\boldsymbol{h}_{1}-\boldsymbol{h}_{0}\right) \ell^{2}+2\left(\boldsymbol{h}_{0}-4 \boldsymbol{h}_{1}+\boldsymbol{h}_{2}\right) \ell^{4} \\
& +\frac{4}{3}\left(3 \boldsymbol{h}_{1}-3 \boldsymbol{h}_{2}-\boldsymbol{h}_{0}+\boldsymbol{h}_{3}\right) \ell^{6}+o\left(\ell^{7}\right) \\
= & 1-\frac{1}{2} \ell^{2}+\frac{1}{6} \ell^{4}-\frac{32}{720} \ell^{6}+o\left(\ell^{7}\right) .
\end{aligned}
$$

We see that these exact series differ slightly in the fourth term, so $F$ and $\boldsymbol{F}$ are not mathematically equal.

\section{5. 'Mean-field' analysis when $q=\frac{1}{2}$}

Gilbert's original 'mean-field' analysis, which was adapted by Mackisack and Miles [7] to the $q=\frac{1}{2}$ rectangular case, involved the rough approximation that ray ends (there being two per seed) were uniformly spread across the plane. With this assumption, it was possible to approximate at time $t$ the expected number of ray ends lying within a small distance $\delta x$ of rays that would block the growth of these ends within the next $\delta t$.

Mackisack and Miles analyzed the $q=\frac{1}{2}$ full model using two quantities: $\boldsymbol{R}(t)$, the expected total length of rays per unit area and $\boldsymbol{G}(t)$, the expected number of growing ends per unit area. Recounting their work, these quantities are related exactly by $\dot{\boldsymbol{R}}=\boldsymbol{G}$, assuming unit growth rate, and heuristically in the full rectangular case by $\dot{\boldsymbol{G}} \approx-\frac{1}{2} \boldsymbol{R} \boldsymbol{G}$, with initial conditions $\boldsymbol{R}(0)=0$ and $\boldsymbol{G}(0)=2 \lambda$. Solving these differential equations, they found that $\boldsymbol{G}(t) \approx 2 \lambda \operatorname{sech}^{2}(t \sqrt{\lambda / 2})$. If $L$ is the final length of a test ray in their full-Gilbert model then

$$
\operatorname{Pr}\{L>\ell\}=\frac{\boldsymbol{G}(\ell)}{\boldsymbol{G}(0)}=\frac{\boldsymbol{G}(\ell)}{2 \lambda} \approx \operatorname{sech}^{2}\left(\ell \sqrt{\frac{\lambda}{2}}\right) .
$$

The expected $L$ when $q=\frac{1}{2}$ is therefore approximated by $\sqrt{2 / \lambda}=1.41421$ at $\lambda=1$. This is not especially close to the value shown in (4). The solution for $\boldsymbol{R}$ was $\boldsymbol{R}(t) \approx$ $2 \sqrt{2 \lambda} \tanh (t \sqrt{\lambda / 2}), t>0$. 
We have modified the analysis in [7] to deal with the $q=\frac{1}{2}$ half-Gilbert model. We put $\dot{G} \approx-\frac{1}{4} R G$ since each of the four directions of growing lines can be blocked by only one other line type. Solving the new equation pair, we find that the number of growing lines per unit area at time $t$ for the half model is $G(t) \approx 2 \lambda \operatorname{sech}^{2}(t \sqrt{\lambda / 4})$. Also, $R(t) \approx 4 \sqrt{\lambda} \tanh (t \sqrt{\lambda / 4}), t>0$. Furthermore, (5) becomes $\operatorname{Pr}\{L>\ell\}=G(\ell) / G(0)=\operatorname{sech}^{2}(\ell \sqrt{\lambda / 4})$. So, setting $\lambda=2$ in the half system and $\lambda=1$ in the full system we obtain identical approximations to the probability density function for the ray length:

$$
f_{2}(\ell) \approx \sqrt{2} \operatorname{sech}^{2} \frac{\ell}{\sqrt{2}} \tanh \frac{\ell}{\sqrt{2}} \approx f_{1}(\ell) .
$$

The expected ray length is $\mathbb{E}(L) \approx \sqrt{2}$.

So we have shown that the mean-field approximations in the two models are equal, when $q=\frac{1}{2}$. Indeed, our analysis for $q \neq \frac{1}{2}$, developed in the next subsection, shows that the two approximations are also equal when $q \neq \frac{1}{2}$.

\subsection{Mean-field analysis when $q \neq \frac{1}{2}$}

When the intensities of $\mathrm{H}$ - and V-type seeds are not equal, the east-growing and southgrowing rays have different length distributions. So a system of four differential equations and four initial values is needed, in the variables (for the half-Gilbert model) $G_{\downarrow}, G_{\rightarrow}, R_{\downarrow}$, and $R_{\rightarrow}$ :

$$
\begin{aligned}
\dot{R}_{\rightarrow}(t) & =G_{\rightarrow}(t), & \dot{G}_{\rightarrow}(t) & \approx-R_{\downarrow}(t) G_{\rightarrow}(t), \\
\dot{R}_{\downarrow}(t) & =G_{\downarrow}(t), & \dot{G}_{\downarrow}(t) & \approx-R_{\rightarrow}(t) G_{\downarrow}(t) .
\end{aligned}
$$

The initial conditions are

$$
R_{\rightarrow}(0)=R_{\downarrow}(0)=0, \quad G_{\rightarrow}(0)=q \lambda, \quad G_{\downarrow}(0)=(1-q) \lambda .
$$

Replacing ' $\approx$ ' with '=' and eliminating $G_{\rightarrow}$ and $G_{\downarrow}$, the differential equations become

$$
\ddot{R}_{\rightarrow}(t)=-R_{\downarrow}(t) \dot{R}_{\rightarrow}(t), \quad \ddot{R}_{\downarrow}(t)=-R_{\rightarrow}(t) \dot{R}_{\downarrow}(t),
$$

augmented by

$$
R_{\rightarrow}(0)=R_{\downarrow}(0)=0, \quad \dot{R}_{\rightarrow}(0)=q \lambda, \quad \dot{R}_{\downarrow}(0)=(1-q) \lambda .
$$

We have only been able to solve this coupled system in series form and, even then, with no general term recognized. Using the abbreviations $Q:=q \lambda$ and $P:=(1-q) \lambda$,

$$
\begin{aligned}
R_{\rightarrow}(t)= & \frac{Q}{1 !} t-\frac{P Q}{3 !} t^{3}+\frac{P Q(3 P+Q)}{5 !} t^{5}-\frac{P Q\left(15 P^{2}+16 P Q+3 Q^{2}\right)}{7 !} t^{7} \\
& +\frac{P Q\left(105 P^{3}+241 P^{2} Q+135 P Q^{2}+15 Q^{3}\right)}{9 !} t^{9}-\cdots
\end{aligned}
$$

with $G_{\rightarrow}(t)$ being $\dot{R}_{\rightarrow}(t)$ (easily calculated from (6)). A MATHEMATICA ${ }^{\circledR}$ routine to compute as many terms as required is available from the authors. For $R_{\downarrow}$ and $G_{\downarrow}$, simply interchange $P$ and $Q$. Note that west-growing rays have results identical to east-growing rays-likewise, north and south results are identical. 
For the full-Gilbert model, the equations are very similar, but cast in terms of the four variates $\boldsymbol{G}_{V}, \boldsymbol{G}_{H}, \boldsymbol{R}_{V}$, and $\boldsymbol{R}_{H}$, that is,

$$
\begin{aligned}
\dot{\boldsymbol{R}}_{H}(t) & =\boldsymbol{G}_{H}(t), & & \dot{\boldsymbol{G}}_{H}(t) \approx-\boldsymbol{R}_{V}(t) \boldsymbol{G}_{H}(t), \\
\dot{\boldsymbol{R}}_{V}(t) & =\boldsymbol{G}_{V}(t), & & \dot{\boldsymbol{G}}_{V}(t) \approx-\boldsymbol{R}_{H}(t) \boldsymbol{G}_{V}(t),
\end{aligned}
$$

with

$$
\boldsymbol{R}_{H}(0)=\boldsymbol{R}_{V}(0)=0, \quad \boldsymbol{G}_{H}(0)=2 q \lambda, \quad \boldsymbol{G}_{V}(0)=2(1-q) \lambda .
$$

This leads to a solution for $\boldsymbol{R}_{H}(t)$ equal to the right-hand side of (6), but with $Q=2 q \lambda$ and $P=2(1-q) \lambda$. Thus, it becomes obvious that $\boldsymbol{R}_{H}(t)$ with $\lambda=1$ equals $R_{\rightarrow}(t)$ with $\lambda=2$, and, likewise, for the other linked pairs of variables. Therefore, when $q \neq \frac{1}{2}$, the two ray length distributions (for $\mathrm{H}$ and $\mathrm{V}$ rays) for the full model having intensity $\lambda$ are equal to the corresponding ray length distributions for the half-Gilbert model with seed intensity $2 \lambda$. All of these entities are, of course, only approximate solutions to the true Gilbert models.

Figure 3 shows that their value as approximations for the full-Gilbert model is quite good, but not nearly as good as the analytic answers adopted from the half-Gilbert model. In Section 5 we provide more of these answers, demonstrating that the half-Gilbert model of Cowan and Ma is encouragingly tractable.

\section{Stopping sets and dead zones in the half-Gilbert model}

It is possible to use the stopping-set concept to find exact expressions for the first, second, and, in principle, higher moments of the ray length in the half-Gilbert model. The balanced case, $q=\frac{1}{2}$, is easier to describe-and that is now our focus. We give some results for the general case in Appendix A.

\subsection{A different construction of stopping sets}

Suppose that a stationary Poisson process of intensity $\lambda$ exists in the plane, with seeds marked either $\mathrm{H}$ (east growing) or V (south growing) with equal probability. In Section 3 we have described how a nest of stopping sets is created when the growth frontier of an expanding domain hits the seeds. For the half-Gilbert model, the seeds that are relevant for an east-growing test ray commencing at $O$ in Figure 2(a) is the shaded region in that figure, or, more precisely, the unbounded octant lying between $y=x$ and $y=0$, with $x \geq 0$ : we call this region the initial live zone.

As before, we start by expanding a domain, an isosceles right-angled triangle in this case (see Figure 6), into the live zone, stopping when it hits the first seed $s_{1}$ whose coordinates relative to $O$ are $\left(x_{1}, y_{1}\right)$. This creates a domain $S_{1}$ with area $E_{1}$ that is exponentially distributed. If $s_{1}$ is $\mathrm{V}$ type then it will provide the ray that blocks the test seed; thus, $L=x_{1}$ and no other seeds need be considered.

Alternatively, if $s_{1}$ is $\mathrm{H}$ type then, instead of growing $S_{1}$ (retaining its shape as an isosceles right-angled triangle and constructing the familiar Zuyev nest of stopping sets), we introduce a significant modification. We remove a part of the live zone: a 'dead zone' labelled $D_{1}$ (see Figure 6) which has now become irrelevant, as we will soon see.

As $S_{1} \cup D_{1}$ has been constructed without drawing upon any information taken from outside $S_{1} \cup D_{1}$, the point process in the remaining region (the new live zone) is still a Poisson process with unchanged intensity given the information within $S_{1} \cup D_{1}$, as explained in Section 3 .

We now grow a trapezium whose left-hand side located at $x=x_{1}$ has length $y=y_{1}$. The trapezium expands until its right-hand side first hits a seed $s_{2}$ (in the new live zone). The stopping set formed is called $S_{2}$. It has an exponentially distributed area $E_{2}$. 


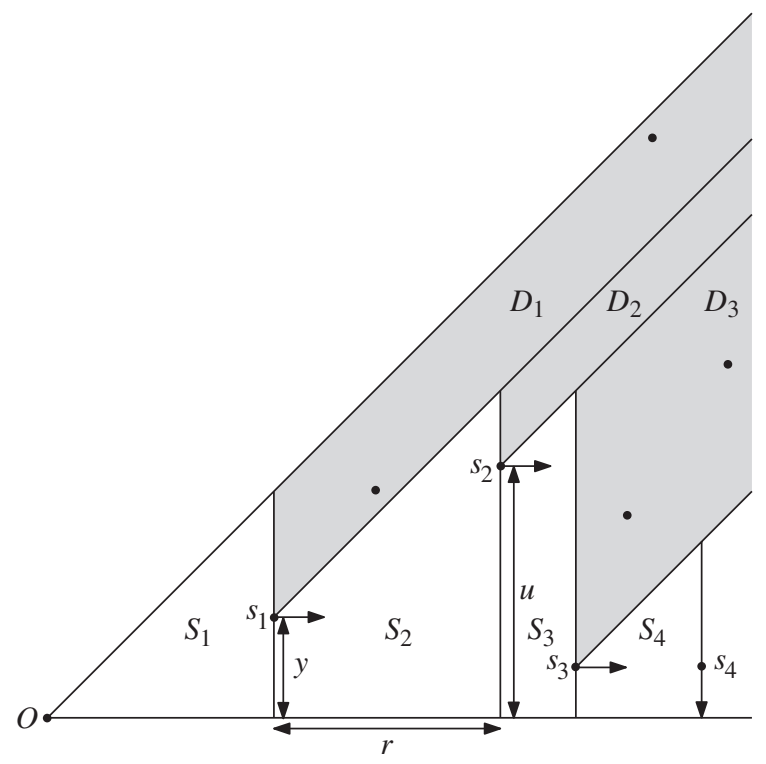

FIGURE 6: Trapezoidal stopping sets and dead zones in the half model.

We proceed in this way, forming a sequence of stopping sets (illustrated in Figure 6) which, unlike those in Section 3, do not form a nest. They do, however, have independent, exponentially distributed areas and are part of a recursive structure which we can exploit. It is also important to note that the first V-type seed will provide the ray which blocks the test ray. Without our introduction of dead zones, a complicated algorithm rather like block would be required to check if a V-type ray actually reaches the path of the test ray.

Remark 4. Dead zone V types will either be blocked by an east-growing ray within the live zone or, if they are not blocked, the test ray must have been intersected at an earlier point. Dead zone $\mathrm{H}$ types can never be in a geometrical position to block live zone $\mathrm{V}$ types. This explains why no seed in dead zones $D_{1}, D_{2}, D_{3}, \ldots$ can influence the distance $L$ travelled by the test seed.

Remark 5. In Remark 2 we mentioned that extraneous randomizations, those not solely dependent on the Poisson point process, might invalidate the key results from the stopping-set theory. There is no such problem here with the stopping set $S_{k}$ itself, but we note that the existence of the $D_{k}, k \geq 1$, depend on an extraneous random feature, namely the $\mathrm{H}$ or $\mathrm{V}$ mark of seed $s_{k}$. This does not invalidate our comment above that the point process in the current live zone outside $S_{k} \cup D_{k}$ is unaffected by the information in $S_{k} \cup D_{k}$. For one thing, the seed marks are independent of each other and of the point process. Furthermore, we stop constructing dead zones only when we have no further need to observe the process at all. So the extraneous random feature is not operative in our analysis.

\subsection{The recursive structure commencing with a generic live zone}

Suppose that we begin observing the process when the live zone has a left boundary of height $y$ and when we are about to construct $S_{n}$. In Figure 6, we illustrate the $n=2$ case. The probability density function for the length, $r$, of $S_{n}$ 's base, conditional on the height $y$ of 
its left boundary, follows from the exponential distribution of $S_{n}$ 's area $E_{n}$. It is, therefore,

$$
f_{R}(r \mid y)=\lambda(r+y) \mathrm{e}^{-\lambda\left(r^{2}+2 r y\right) / 2}, \quad r>0 .
$$

Furthermore, the left boundary height, $u$ say, of the next set $S_{n+1}$ is uniformly distributed on the growth frontier of $S_{n}$ (a fact noted in the text following Remark 2), so has the following probability density function conditional upon $y$ and $r$ :

$$
f_{U}(u \mid y, r)=\frac{1}{r+y}, \quad 0<u<r+y .
$$

If the stopping seed $s_{n}$ for set $S_{n}$ is $\mathrm{V}$ type then its south ray will be the first to intersect the test ray and the process ends. Otherwise, another dead zone is created and further trapezoidal stopping sets are formed until a $\mathrm{V}$ type is met.

Let $X$ be the random variable equal to the horizontal distance covered by stopping sets until the process comes to an end. Denote the density function of $X$ conditional on $y$ by $g(x \mid y)$. Then

$$
\begin{aligned}
g(x \mid y)= & \frac{\lambda}{2}(x+y) \mathrm{e}^{-\lambda\left(x^{2}+2 x y\right) / 2} \\
& +\frac{1}{2} \int_{0}^{\infty}\left(\int_{0}^{r+y} g(x-r \mid u) f_{U}(u \mid y, r) \mathrm{d} u\right) f_{R}(r \mid y) \mathrm{d} r \\
= & \frac{\lambda}{2}\left[(x+y) \mathrm{e}^{-\lambda\left(x^{2}+2 x y\right) / 2}+\int_{0}^{\infty} \mathrm{e}^{-\lambda\left(r^{2}+2 r y\right) / 2}\left(\int_{0}^{r+y} g(x-r \mid u) \mathrm{d} u\right) \mathrm{d} r\right],
\end{aligned}
$$

where $g(x \mid y)=0$ if $x<0$. In (7) the first term accounts for the case where the first seed is $\mathrm{V}$ type and the second term for the case where it is $\mathrm{H}$ type, and the process is effectively restarted with a different boundary condition $y$ (now labeled $u$ in Figure 6) having already covered some horizontal distance $r$. This second term conditions further on $r$ and $u$.

We have set $q=\frac{1}{2}$, but the analysis leading to (7) can be carried out for general $q$, producing a more complicated result. Note that the ray length probability density function is $g(x \mid 0)$.

We define the moments of the conditional density by

$$
\mu_{n}(y)=\int_{0}^{\infty} x^{n} g(x \mid y) \mathrm{d} x .
$$

As mentioned before, we will here compute $\mathbb{E}(L)=\mu_{1}(0)$ and $\mathbb{E}\left(L^{2}\right)=\mu_{2}(0)$, which, from (7), satisfy

$$
\begin{aligned}
& \mu_{1}(0)=\sqrt{\frac{\pi}{2 \lambda}}\left[1+\frac{\lambda}{2} \int_{0}^{\infty} \operatorname{erfc}\left(\sqrt{\frac{\lambda}{2}} u\right) \mu_{1}(u) \mathrm{d} u\right], \\
& \mu_{2}(0)=\frac{2}{\lambda}+\frac{\lambda}{2} \sqrt{\frac{\pi}{2 \lambda}} \int_{0}^{\infty} \operatorname{erfc}\left(\sqrt{\frac{\lambda}{2}} u\right) \mu_{2}(u) \mathrm{d} u+\int_{0}^{\infty} \mathrm{e}^{-\lambda u^{2} / 2} \mu_{1}(u) \mathrm{d} u .
\end{aligned}
$$

Our strategy is to find $\mu_{1}(y)$ and $\mu_{2}(y)$ up to an arbitrary constant, and then to determine the constant using (8) and (9). The first part of this process is most easily achieved by making use of the moment generating function

$$
M_{t}(y)=\int_{0}^{\infty} \mathrm{e}^{t x} g(x \mid y) \mathrm{d} x
$$


which, from (7), satisfies

$$
\begin{aligned}
M_{t}(y)=\frac{1}{2}+\frac{1}{2} \sqrt{\frac{\pi}{2 \lambda}} \mathrm{e}^{(\lambda y-t)^{2} / 2 \lambda}\left\{\operatorname{erfc}\left(\frac{\lambda y-t}{\sqrt{2 \lambda}}\right)\left[t+\lambda \int_{0}^{y} M_{t}(u) \mathrm{d} u\right]\right. \\
\left.+\lambda \int_{y}^{\infty} \operatorname{erfc}\left(\frac{\lambda u-t}{\sqrt{2 \lambda}}\right) M_{t}(u) \mathrm{d} u\right\} .
\end{aligned}
$$

This integral equation can be reduced to the differential equation

$$
\frac{\mathrm{d}^{2} M_{t}}{\mathrm{~d} y^{2}}-(\lambda y-t) \frac{\mathrm{d} M_{t}}{\mathrm{~d} y}-\frac{\lambda}{2} M_{t}=-\frac{\lambda}{2} .
$$

Expressing the left-hand side as a series in $t$, and collecting coefficients of $t$ and $t^{2}$, we obtain differential equations satisfied by $\mu_{1}(y)$ and $\mu_{2}(y)$ :

$$
\begin{gathered}
\mu_{1}^{\prime \prime}(y)-\lambda y \mu_{1}^{\prime}(y)-\frac{\lambda}{2} \mu_{1}(y)=0, \\
\mu_{2}^{\prime \prime}(y)-\lambda y \mu_{2}^{\prime}(y)-\frac{\lambda}{2} \mu_{2}(y)=-2 \mu_{1}^{\prime}(y) .
\end{gathered}
$$

Clearly, we must solve for $\mu_{1}(y)$ first.

\subsection{The first conditional moment}

Making the change of variable $z=(\lambda / 2)^{1 / 2} y$ in (10) we obtain

$$
\frac{\mathrm{d}^{2} \mu_{1}}{\mathrm{~d} z^{2}}-2 z \frac{\mathrm{d} \mu_{1}}{\mathrm{~d} z}-\mu_{1}=0 .
$$

If the coefficient of $\mu_{1}$ were a positive multiple of two, this would be Hermite's equation, solved by Hermite polynomials. Since this is not the case, we seek a series solution [8]

$$
\mu_{1}(y(z))=\sum_{n=0}^{\infty} a_{n} z^{n}
$$

and obtain the recurrence relation

$$
a_{n+2}=\frac{2 n+1}{(n+1)(n+2)} a_{n} .
$$

This leads to the general solution

$$
\mu_{1}(y(z))=a_{0} M\left(\frac{1}{4}, \frac{1}{2}, z^{2}\right)+a_{1} z M\left(\frac{3}{4}, \frac{3}{2}, z^{2}\right),
$$

where $M$ is Kummer's function [1]:

$$
M(a, b, z)=\sum_{n=0}^{\infty} \frac{(a)_{n} z^{n}}{(b)_{n} n !} .
$$

Here we have used the Pochhammer symbol, defined by

$$
(a)_{n}=a(a+1)(a+2) \cdots(a+n-1), \quad(a)_{0}=1 .
$$


Kummer's functions diverge as $z \rightarrow \infty$, but we know that $\mu_{1}(y(z)) \rightarrow 0$ in that limit. This apparent paradox is resolved by noting that the two independent parts of the solution may be combined to form a Kummer function of the second kind [1], defined by

$$
U(a, b, z)=\frac{\pi}{\sin \pi b}\left[\frac{M(a, b, z)}{\Gamma(1+a-b) \Gamma(b)}-z^{1-b} \frac{M(1+a-b, 2-b, z)}{\Gamma(a) \Gamma(2-b)}\right],
$$

which tends to 0 as $z \rightarrow \infty$. In terms of this function, the general solution is

$$
\mu_{1}(y(z))=A M\left(\frac{1}{4}, \frac{1}{2}, z^{2}\right)+B U\left(\frac{1}{4}, \frac{1}{2}, z^{2}\right) .
$$

It must be the case that $A=0$ in order to capture the right asymptotic behaviour, so, restoring the original variable $y$, the conditional moment must have the form

$$
\mu_{1}(y)=B U\left(\frac{1}{4}, \frac{1}{2}, \frac{\lambda}{2} y^{2}\right) .
$$

It now remains to compute $B$. We do this by substituting (12) into (8). Making use of the result

$$
\int_{0}^{\infty} \operatorname{erfc}(u) U\left(\frac{1}{4}, \frac{1}{2}, u^{2}\right) \mathrm{d} u=\frac{\sqrt{2}}{\pi}\left[\Gamma\left(\frac{1}{4}\right)-\sqrt{\pi} \Gamma\left(\frac{3}{4}\right)\right],
$$

together with $\Gamma\left(\frac{1}{4}\right) \Gamma\left(\frac{3}{4}\right)=\sqrt{2} \pi$ and $U\left(\frac{1}{4}, \frac{1}{2}, 0\right)=\sqrt{\pi} / \Gamma\left(\frac{3}{4}\right)$, we find that

$$
B=\frac{\sqrt{\pi}}{\sqrt{\lambda} \Gamma(3 / 4)} .
$$

We have now found $\mu_{1}(y)$, which gives us a compact analytic expression for the expected ray length:

$$
\mathbb{E}(L)=\mu_{1}(0)=\frac{\sqrt{\pi}}{\sqrt{\lambda} \Gamma(3 / 4)} U\left(\frac{1}{4}, \frac{1}{2}, 0\right)=\frac{\pi}{\sqrt{\lambda}(\Gamma(3 / 4))^{2}} \approx \frac{2.0920992}{\sqrt{\lambda}} .
$$

For comparison, using the first 200 coefficients from Cowan and Ma's recurrence, we obtain $\mathbb{E}(L) \approx 2.0920987$ when $\lambda=1$. As we discovered earlier, when $\lambda=2$, the half model provides an approximation to the full model, having similar but simplified blocking effects and identical mean field behaviour. For this choice of $\lambda$, we obtain the exact half model result $\mathbb{E}(L)=1.479337560$ to seven decimal places, which differs from the accurate full model result $(1.467535)$ by $0.7 \%$. Compared with the mean-field prediction, $\mathbb{E}(L) \approx \sqrt{2}$, which differs from the full model by $3.6 \%$, this is a much closer approximation.

\subsection{The second conditional moment}

As for the calculation of $\mu_{1}$, we make the change of variable $z=(\lambda / 2)^{1 / 2} y$, but this time in (11), obtaining

$$
\frac{\mathrm{d}^{2} \mu_{2}}{\mathrm{~d} z^{2}}-2 z \frac{\mathrm{d} \mu_{2}}{\mathrm{~d} z}-\mu_{2}=\frac{\sqrt{2 \pi} z}{\lambda \Gamma(3 / 4)} U\left(\frac{5}{4}, \frac{3}{2}, z^{2}\right),
$$

where we have used the differential property [1] $U^{\prime}(a, b, z)=-a U(a+1, b+1, z)$. We know the homogeneous part of the general solution to (11), so it remains to find a particular solution. We do this using variation of parameters, and begin by making the definitions

$$
f_{1}(z)=M\left(\frac{1}{4}, \frac{1}{2}, z^{2}\right), \quad f_{2}(z)=U\left(\frac{1}{4}, \frac{1}{2}, z^{2}\right) .
$$


The function $M$ has the differential property [1] $M^{\prime}(a, b, z)=(a / b) M(a+1, b+1, z)$, which allows us to compute the Wronskian

$$
\begin{aligned}
W(z) & =f_{1}(z) f_{2}^{\prime}(z)-f_{2}(z) f_{1}^{\prime}(z) \\
& =-\frac{1}{2} Z\left[M\left(\frac{1}{4}, \frac{1}{2}, z^{2}\right) U\left(\frac{5}{4}, \frac{3}{2}, z^{2}\right)+2 U\left(\frac{1}{4}, \frac{1}{2}, z^{2}\right) M\left(\frac{5}{4}, \frac{3}{2}, z^{2}\right)\right]
\end{aligned}
$$

We now define

$$
G(z, t)=\frac{f_{2}(z) f_{1}(t)-f_{1}(z) f_{2}(t)}{W(t)}
$$

in terms of which the particular integral is

$$
f_{p}(z)=-\frac{\sqrt{2 \pi}}{\lambda \Gamma(3 / 4)} \int_{z}^{\infty} G(z, t) t U\left(\frac{5}{4}, \frac{3}{2}, t^{2}\right) \mathrm{d} t .
$$

Discarding the divergent part of the solution, and restoring $y$, we have

$$
\mu_{2}(y)=C U\left(\frac{1}{4}, \frac{1}{2}, \frac{\lambda}{2} y^{2}\right)-\frac{\sqrt{2 \pi}}{\lambda \Gamma(3 / 4)} \int_{z(y)}^{\infty} G(z(y), t) t U\left(\frac{5}{4}, \frac{3}{2}, t^{2}\right) \mathrm{d} t,
$$

where $C$ is an as yet undetermined constant. We find it by substituting our expression for $\mu_{2}(y)$ into (9). Making use of the numerical integral

$$
K=-\int_{0}^{\infty} \operatorname{erfc}(z)\left[\int_{z}^{\infty} G(z, t) t U\left(\frac{5}{4}, \frac{3}{2}, t^{2}\right) \mathrm{d} t\right] \mathrm{d} z=0.343146,
$$

we find that

$$
C=\frac{1}{\Gamma(3 / 4) \lambda}\left(\frac{\pi K}{\Gamma(3 / 4)}+2 \sqrt{2}\right)
$$

Noting also that $f_{p}(0)=2 / \lambda$ we have the final result:

$$
\begin{aligned}
\mathbb{E}\left(L^{2}\right) & =\mu_{2}(0) \\
& =\frac{1}{\Gamma(3 / 4) \lambda}\left(\frac{\pi K}{\Gamma(3 / 4)}+2 \sqrt{2}\right) U\left(\frac{1}{4}, \frac{1}{2}, 0\right)+f_{p}(0) \\
& =\frac{\pi^{3 / 2} K+2 \Gamma(3 / 4)\left(\sqrt{2 \pi}+\Gamma(3 / 4)^{2}\right)}{\lambda \Gamma(3 / 4)^{3}} \\
& \approx \frac{6.37688}{\lambda} .
\end{aligned}
$$

For comparison, using the first 200 coefficients from the Cowan-Ma recurrence, we obtain $\mathbb{E}(L) \approx 6.37686$ when $\lambda=1$.

\section{Concluding comment}

Gilbert's tessellation is notoriously difficult to analyze, and even the rectangular version studied by Mackisack and Miles remains entirely without analytical results. In this paper we have shown that the simplified rectangular model of Cowan and Ma, with only half of the blocking rules of the Mackisack and Miles model, has a number of tractable properties. As such, it is the only Gilbert-style model, we believe, which has yielded any analytic results. 


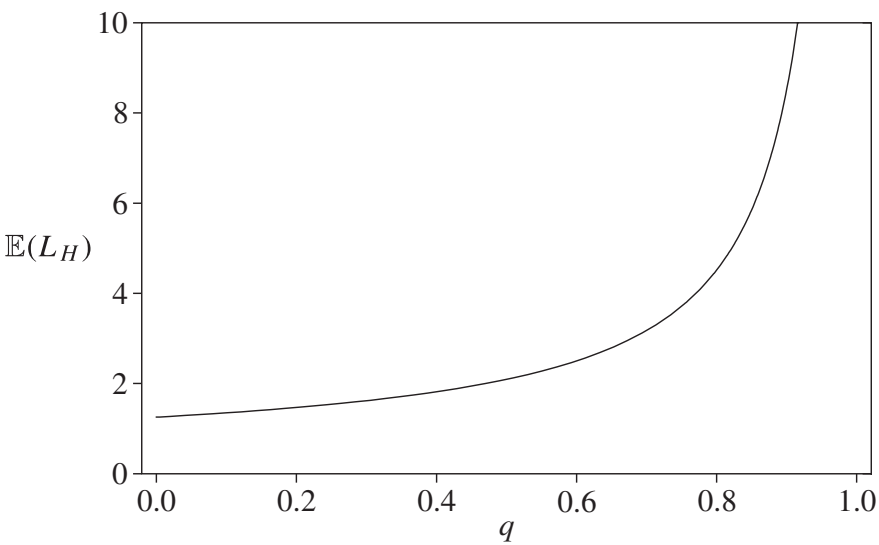

FIGURE 7: Expected horizontal length in the half model as a function of $q$, the proportion of horizontal rays. The seed density is $\lambda=1$.

\section{Appendix A. Expected length in the half model when $q \neq \frac{1}{2}$}

If $q$ is the proportion of seeds growing horizontally in the half model then (7) becomes

$$
\begin{aligned}
g(x \mid y)= & (1-q) \lambda(x+y) \mathrm{e}^{-\lambda\left(x^{2}+2 x y\right) / 2} \\
& +q \lambda \int_{0}^{\infty} \mathrm{e}^{-\lambda\left(r^{2}+2 r y\right) / 2}\left[\int_{0}^{r+y} g(x-r \mid u) \mathrm{d} u\right] \mathrm{d} r .
\end{aligned}
$$

The first moment of $g(x \mid 0)$ may be found by similar methods to those employed in the $q=\frac{1}{2}$ case. The expected length of a horizontal ray is found to be

$$
\mathbb{E}\left(L_{H}\right)=\sqrt{\frac{\pi}{\lambda}}\left[\sqrt{2}-\frac{1}{2^{q+1 / 2} \pi \Gamma(1-q)} q \Gamma\left(1-\frac{q}{2}\right) G_{3,3}^{2,3}\left(\begin{array}{l|l}
1 & 0,1 / 2,(q+1) / 2 \\
0,1 / 2,-1 / 2
\end{array}\right)\right]^{-1}
$$

where $G$ is Meijer's G-function [6]. In Figure 7 we plot the function $\mathbb{E}\left(L_{H}\right)$ for the $\lambda=1$ case.

\section{Acknowledgements}

We thank the anonymous referees for their helpful comments.

\section{References}

[1] Abramowitz, M. And Stegun, I. A. (1970). Handbook of Mathematical Functions. Dover Publications, New York.

[2] Burridge, J. (2010). Simulation of the rectangular Gilbert tessellation. Unpublished Tech. Rep.

[3] Cowan, R. and Ma, I. S. W. (2002). Solving a simplified version of the Gilbert tessellation. Available at http://www.maths.usyd.edu.au/u/richardc/unsolved.html.

[4] Cowan, R., Quine, M. And Zuyev, S. (2003). Decomposition of gamma-distributed domains constructed from Poisson point processes. Adv. Appl. Prob. 35, 56-69.

[5] Gilbert, E. N. (1967). Surface films of needle-shaped crystals. In Applications of Undergraduate Mathematics in Engineering, ed. B. Noble, Macmillan, pp. 329-346.

[6] Gradshteyn, I. S. And Ryzhik, I. M. (2007). Table of Integrals, Series, and Products, 7th edn. Academic Press, Amsterdam. 
[7] Mackisack, M. S. and Miles, R. E. (1996). Homogeneous rectangular tessellations. Adv. Appl. Prob. 28, 993-1013.

[8] Mathews, J. And Walker, R. L. (1973). Mathematical Methods of Physics. Addison-Wesley.

[9] Schreiber, T. and Soja, N. (2011). Limit theory for planar Gilbert tessellations. Prob. Math. Statist. 31, 149-160.

[10] ZuYev, S. (1999). Stopping sets: gamma-type results and hitting properties. Adv. Appl. Prob. 31, 355-366. 